\title{
Sangramento uterino anormal e o impacto na qualidade de vida de mulheres atendidas em unidade especializada do Amazonas
}

\author{
Abnormal uterine bleeding and the impact on the quality of life of women treated at a \\ specialized unit in Amazonas
Sangrado uterino anormal e impacto en la calidad de vida de mujeres atendidas en una unidad especializada en Amazonas

Camila Peixoto Maia ${ }^{1 *}$, Heliamara Ferreira Maia Teles ${ }^{1}$, Amanda Peixoto Maia ${ }^{2}$, Filipe Peixoto Maia $^{2}$, Laura Maria Araújo Esper².

\begin{abstract}
RESUMO
Objetivo: Analisar o perfil epidemiológico e o impacto do sangramento uterino anormal (SUA) na qualidade de vida de pacientes com este sintoma, em ambulatório de ginecologia da cidade de Manaus, de junho a dezembro de 2020. Métodos: Estudo observacional, transversal, prospectivo; dados referentes a identificação, perfil epidemiológico e caracterização do sangramento obtidos por questionário fechado foram tabulados, analisados no SPSS e organizados em tabelas e gráficos no Excel. Resultados: Foram atendidas 60 pacientes com SUA, a maioria entre 40 e 50 anos de idade (45\%), escolaridade de nível médio (50\%), de cor parda $(78,3 \%)$, natural e procedente de Manaus (58,3\% e 86,7\%) com renda familiar média de 1.925 reais. O tempo de evolução dos sintomas foi em média de 11,5 meses. $50 \%$ definiram a alteração do padrão menstrual como aumento do fluxo. $56 \%$ classificaram sua qualidade de vida entre regular e muito ruim. $46,7 \%$ referiram impacto do sangramento no dia a dia e 58,3\%, interferência na vida sexual. Conclusão: O SUA interfere negativamente na qualidade de vida feminina com maior ênfase na vida sexual e social. A maioria das mulheres com SUA apresentam aumento do fluxo menstrual. É necessário o desenvolvimento de abordagem individualizada e integral para o tratamento resolutivo.
\end{abstract}

Palavras-chave: Sangramento uterino anormal, Qualidade de vida, Saúde da mulher.

\begin{abstract}
Objective: To analyze the epidemiological profile and the impact of abnormal uterine bleeding (AUB) on the quality of life of patients seen with this symptom, at a gynecology outpatient clinic, in the city of Manaus, from June to December 2020. Methods: Observational, cross-sectional and prospective study; data regarding the identification, epidemiological profile and characterization of bleeding obtained by a closed questionnaire was tabulated, analyzed in SPSS and organized in tables and graphs in Excel. Results: 60 patients with AUB were attended, the majority between 40 and 50 years of age (45\%), finished middle schooling (50\%), ethnically brown (78.3\%), born and hailing from Manaus (58,3\% and $86.7 \%$ ) with an average family income of $R \$ 1,925$. Symptoms evolved over an average of 11.5 months. $50 \%$ classified the change in their menstrual pattern as increased flow. $56 \%$ rated their quality of life between fair and very poor. $46.7 \%$ reported the impact of bleeding on a daily basis and $58.3 \%$ reported interference in sexual life. Conclusion: AUB interferes negatively in the quality of life of women with a greater emphasis on sexual and social life. Most women with AUB have increased menstrual flow. It is necessary to develop an individualized and comprehensive approach to resolutive treatment.
\end{abstract}

Key words: Abnormal uterine bleeding, Quality of life, Women's health.

\footnotetext{
${ }_{1}$ Universidade do Estado do Amazonas (UEA), Manaus - AM. *E-mail: camilamaia.com@gmail.com

2 Universidade Nilton Lins (UNL), Manaus - AM.
}

SUBMETIDO EM: 4/2021

ACEITO EM: 5/2021

PUBLICADO EM: 5/2021 


\section{RESUMEN}

Objetivo: Analizar el perfil epidemiológico y el impacto del sangrado uterino anormal (SUA) en la calidad de vida de las pacientes con este síntoma, en ambulatorio de ginecología en la ciudad de Manaus, de junio a diciembre de 2020. Métodos: Estudio observacional, transversal y prospectivo; los datos sobre la identificación, el perfil epidemiológico y la caracterización de la hemorragia obtenidos mediante un cuestionario cerrado fueron tabulados, analizados en SPSS y organizados en tablas y gráficos en Excel. Resultados: se atendieron 60 pacientes con SUA, la mayoría entre 40 y 50 años (45\%), secundaria terminada $(50 \%)$, de etnia morena $(78,3 \%)$, nacidos y oriundos de Manaos ( $58,3 \%$ y $86,7 \%)$. \%) con un ingreso familiar promedio de R $\$ 1.925$. Los síntomas evolucionaron durante un promedio de 11,5 meses. El $50 \%$ clasificó el cambio en su patrón menstrual como aumento del flujo. El 56\% calificó su calidad de vida entre regular y muy mala. El 46,7\% informó el impacto del sangrado a diario y el 58,3\% informó interferencia en la vida sexual. Conclusión: EI SUA interfiere negativamente en la calidad de vida de las mujeres con un mayor énfasis en la vida sexual y social. La mayoría de las mujeres con SUA presentan un aumento del flujo menstrual. Es necesario el desarrollo de un enfoque individualizado e integral para un tratamiento resolutivo.

Palabras clave: Sangrado uterino anormal, Calidad de vida, Salud de la mujer.

\section{INTRODUÇÃO}

O Sangramento Uterino Anormal (SUA) é um problema clínico que está entre as principais queixas ginecológicas, sendo responsável por $30 \%$ das consultas ao ginecologista no caso de mulheres na premenopausa e de $70 \%$ das mulheres na perimenopausa e pós-menopausa afetando todas as idades, acometendo cerca de $10 \%$ da população em idade reprodutiva e interferindo diretamente em aspectos físicos, sexuais, profissionais, sociais com consequências desagradáveis à qualidade de vida das pacientes. Essas interferências tendem a se desdobrar em outras problemáticas notadamente no âmbito emocional, por induzirem condições psicossociais desagradáveis, decorrentes das limitações impostas pelo SUA, principalmente em mulheres que apresentam essa condição por longo tempo e de forma mais severa (ARLINDO EM, et al., 2018).

SUA é definido como alterações de características do padrão de sangramento como aumento do volume, duração ou frequência, ocorrendo de forma crônica ou aguda. Por haver grande dificuldade na avaliação objetiva da perda sanguínea, a definição associada à interferência na qualidade de vida substitui a quantificação da perda sanguínea como critério diagnóstico (BENETTI-PINTO CL, et al., 2017).

A avaliação diagnóstica de pacientes com SUA é complexa. Devem ser investigados sintomas associados ao sangramento como dismenorreia, dispareunia, dor abdominal, febre, corrimento vaginal, assim como o padrão normal de sangramento, individualizando a paciente, avaliando a evolução e o padrão do fluxo atual, as características do sangramento, além de medicações concomitantes, tratamentos realizados e cirurgias prévias (KAUNITZ AM, 2021).

É importante considerar a idade da mulher, pois permite um direcionamento clínico para as causas mais prevalentes por faixa etária. Em crianças, por exemplo, o sangramento pode estar relacionado a traumas, infecções vaginais ou violência sexual. Já no extremo da vida reprodutiva há predomínio do sangramento disfuncional, seguido das neoplasias. A possibilidade de gravidez sempre deve ser descartada em pacientes na menacme (FRASER IS, 2018).

O exame físico minucioso é fundamental na propedêutica de sangramento anormal, sendo imprescindíveis o exame especular e o toque bimanual. É importante verificar se há lesões perineais, vaginais ou no colo. Exames complementares e de imagem também auxiliam na investigação diagnóstica conforme a suspeita clínica (SINGH S, et al., 2018).

A Federação Internacional de Ginecologia Obstetrícia (FIGO) propôs, em 2011, o sistema de classificação etiológica de SUA a fim de evitar termos inespecíficos. A classificação PALM-COEIN procura sistematizar o diagnóstico e tratamento dessa condição, dividindo as causas em estruturais ( $P$ - pólipos, A- adenomiose, L- 
leiomiomas, M- malignidade e hiperplasia) e não estruturais (C- coagulopatia, O- disfunção ovariana, Edisfunção endometrial, I- iatrogênica, N- não classificada) (MUNRO M, et al., 2011).

Dentre as causas estruturais do SUA estão os (1) pólipos que se apresentam como projeções endometriais acometendo até $30 \%$ das mulheres com sangramento anormal, sendo outros sintomas comuns a dismenorreia secundária e a infertilidade; (2) adenomiose definida pela presença de tecido endometrial entre as fibras do miométrio levando a aumento do volume uterino, podendo evoluir com dor pélvica crônica, infertilidade, dispareunia e dismenorreia, presente em $20 \%$ das mulheres; (3) leiomiomas que são tumorações benignas responsáveis por aproximadamente um terço das histerectomias realizadas, com a sintomatologia variando de acordo com o tamanho e a localização do tumor, e (4) neoplasias podendo destacar, dentre as que cursam com SUA, o câncer de colo uterino e o câncer de endométrio (KAUNITZ AM, 2021).

Nas causas não estruturais as coagulopatias mais associadas ao sangramento são Doença de Von Willebrand e Purpura Trombocitopenica Idiopática. Já a disfunção ovariana mais prevalente na menacme é a Síndrome dos Ovários Policísticos (KAUNITZ AM, 2021).

O SUA agudo ou crônico, é uma condição desafiadora para a equipe de saúde podendo evoluir para quadros de diferentes graus de anemia, desequilíbrio hemodinâmico e choque hipovolêmico nos casos mais graves, por isso é essencial estabelecer o tratamento oportuno de acordo com sua etiologia, se pontual ou prolongado, medicamentoso ou cirúrgico, podendo ser necessária associação entre as modalidades terapêuticas (SILVA NK, 2019).

O tratamento medicamentoso se divide principalmente entre hormonal por meio de anticoncepcionais orais combinados, progestágenos isolados, análogos do GnRH (Hormônio Liberador de Gonadotrofina) dentre outros; e tratamento não hormonal com ácido tranexâmico e AINES (Anti-inflamatórios Não Esteroidais). Dentre as terapias cirúrgicas disponíveis temos a histerectomia quando não há desejo de manutenção uterina e a polipectomia, miomectomia, ablação endometrial e embolização das artérias uterinas com preservação de fertilidade (BENETTI-PINTO CL, et al., 2017).

De forma geral, o SUA pode afetar a saúde, a sensação de bem-estar, a produtividade profissional e qualidade de vida como um todo, porque o equilíbrio emocional decorre diretamente do bem-estar geral, enquanto a qualidade de vida está associada a aspectos como moradia, acesso a bens e serviços, lazer, vida sexual e profissional (MACHADO WL e BANDEIRA DR, 2012).

O SUA tem impacto direto na qualidade de vida das pacientes por estar associada a custos com medicações, internações e procedimentos cirúrgicos, e com o afastamento de atividades laborais devido à debilidade física que pode determinar, além de repercussões fisiológicas diversas (FILHO ALS, 2015). Portanto, essa é uma condição patológica que necessita ter prioridade no âmbito da saúde da mulher, considerando que acarreta diversas alterações na rotina da paciente (trocas constantes de absorventes, desconforto, limitação de atividades, entre outros aspectos que concorrem para a depreciação da qualidade de vida) (YELA DA e BENETTI-PINTO CL, 2018).

Faz-se necessário observarmos que a percepção do que seja qualidade de vida é uma representação sociocultural, portanto, subjetiva e dependente de fatores intrínsecos a cada estamento social, não sendo possível elencarmos uma definição única que abranja todo o espectro de características que a constituem. Nesta pesquisa circunscrevemos este amplo conceito ao estado de saúde física e emocional que propicia a plena atuação profissional, sexual e social (AGUAS F, et al., 2019).

Considerando a carência por estudos que abordem a temática na cidade de Manaus o objetivo deste trabalho foi analisar o impacto do sangramento uterino anormal na qualidade de vida das pacientes atendidas em unidade especializada do Amazonas.

\section{MÉTODOS}

Estudo observacional, transversal, prospectivo com dados obtidos por meio de questionário fechado aplicado às pacientes atendidas em ambulatório de ginecologia da cidade de Manaus, no período de junho a 
dezembro de 2020. A amostra foi calculada com base na população esperada para seis meses (de janeiro a junho de 2019), totalizando uma amostra mínima de 60 pacientes.

Foram incluídas na pesquisa as pacientes entre 18 e 55 anos com queixa de sangramento uterino anormal. Foram excluídos os casos de pacientes indígenas, gestantes, mulheres menores 18 anos de idade ou que não preencheram o questionário adequadamente, ou ainda as que não assinaram o termo de consentimento livre e esclarecido (TCLE).

O estudo foi realizado por meio de variáveis quantitativas e qualitativas agrupadas nas seguintes categorias: Dados de identificação e perfil epidemiológico; histórico ginecológico; caracterização do sangramento e impactos do sangramento nas atividades diárias. As informações foram coletadas por meio do preenchimento de questionário específico após aplicação do termo de consentimento livre e esclarecido.

Os dados foram apresentados por meio de gráficos e tabelas, onde se calculou as frequências absolutas simples e relativas para as diferentes categorias em estudo. $\mathrm{Na}$ análise dos dados quantitativos, quando aceita a hipótese de normalidade por meio do teste de Shapiro-Wilk, foi calculada as seguintes estatísticas descritivas, a média e o Desvio-padrão (Dp), no entanto, quando rejeitada a hipótese de normalidade, foi calculada a mediana. O software utilizado na análise das informações coletadas foi o programa Epi Info versão 7.2 para Windows que é desenvolvido e distribuído gratuitamente pelo Centro de Prevenção e Controle de Doenças Norte-Americano (CDC) e o nível de significância fixado no teste de normalidade foi de 5\% (VIEIRA $S, 2004)$.

Este estudo foi submetido ao Comitê de Ética em Pesquisa envolvendo seres humanos da Universidade do Estado do Amazonas segundo CAAE 28789220.8.0000.5016, conforme parecer número 3.990.190, emitido em 24 de abril de 2020.

\section{RESULTADOS}

No período de junho a dezembro do ano de 2020, 60 pacientes atendidas em ambulatório de ginecologia da cidade de Manaus, com queixa de sangramento uterino anormal preencheram o questionário referente a essa pesquisa. Desse instrumento foram obtidos os dados que passamos a apresentar.

O perfil epidemiológico elaborado indicou que a maioria das pacientes pertencia à faixa etária entre $40 \mathrm{e}$ 50 anos de idade (45\%), possuindo escolaridade de nível médio $(50 \%)$, considerando-se de cor parda (78,3\%), sendo natural e procedente da cidade de Manaus (58,3\% e 86,7\%); também foi entrevisto que fazem parte de lares com renda familiar média de 1.925 reais (Tabela 1). 
Tabela 1 - Distribuição segundo os dados demográficos e socioeconômicos das mulheres com SUA atendidas em ambulatório de ginecologia da cidade de Manaus.

\begin{tabular}{|c|c|c|}
\hline Variáveis $(n=60)$ & Frequência absoluta simples & $\%$ \\
\hline \multicolumn{3}{|l|}{ Idade } \\
\hline $18 \mid---30$ & 12 & 20,0 \\
\hline $30 \mid---40$ & 15 & 25,0 \\
\hline $40 \mid---50$ & 27 & 45,0 \\
\hline $50|--| 55$ & 6 & 10,0 \\
\hline Mediana & 40 & - \\
\hline \multicolumn{3}{|l|}{ Naturalidade } \\
\hline Manaus & 35 & 58,3 \\
\hline Interior do Estado & 23 & 38,3 \\
\hline Outros Estados & 2 & 3,3 \\
\hline \multicolumn{3}{|l|}{ Procedência } \\
\hline Manaus & 52 & 86,7 \\
\hline Interior do Estado & 8 & 13,3 \\
\hline \multicolumn{3}{|l|}{ Escolaridade } \\
\hline Não alfabetizado & 1 & 1,7 \\
\hline Ensino fundamental & 13 & 21,7 \\
\hline Ensino médio & 30 & 50,0 \\
\hline Ensino superior & 16 & 26,6 \\
\hline \multicolumn{3}{|l|}{ Profissão } \\
\hline Dona de casa & 17 & 38,3 \\
\hline Autônoma & 14 & 23,3 \\
\hline Funcionária pública & 7 & 11,7 \\
\hline Estudante & 6 & 10,0 \\
\hline Outras & 16 & 26,7 \\
\hline \multicolumn{3}{|l|}{ Raça } \\
\hline Branca & 12 & 20,0 \\
\hline Parda & 47 & 78,3 \\
\hline Outra & 1 & 1,7 \\
\hline Fuma & 4 & 6,7 \\
\hline Ingere bebida alcoólica & 21 & 35,0 \\
\hline Pratica atividade física & 31 & 51,7 \\
\hline \multicolumn{3}{|l|}{ Renda familiar } \\
\hline Mediana & 1925 & - \\
\hline Mínimo - Máximo & $446-30000$ & - \\
\hline
\end{tabular}

Fonte: Maia CP, et al., 2021.

A investigação da história clínica ginecológica das pacientes participantes da pesquisa resultou nos seguintes dados: 71,7\% apresentaram menarca após os 12 anos de idade; 76,7\% relataram a sexarca após os 15 anos - comportamento sexual muito comum entre as adolescentes da região. Até o momento da pesquisa, $40 \%$ das pacientes participantes desta relataram terem tido entre 2 e 5 parceiros sexuais. A maioria destas mulheres eram multíparas com mais de 3 gestações (51,7\%). 31,7\% das pacientes tiveram parto por cesariana e $58,3 \%$ referiram pelo menos um parto por via vaginal. Da investigação clínica ginecológica o dado que mais se destacou foi a alta média de tempo decorrido desde o início dos sintomas de sangramento anormal, registrando 11,5 meses (Gráfico 1). 
Gráfico 1 - Distribuição segundo o tempo de início dos sintomas nas mulheres com SUA atendidas em ambulatório de ginecologia da cidade de Manaus.

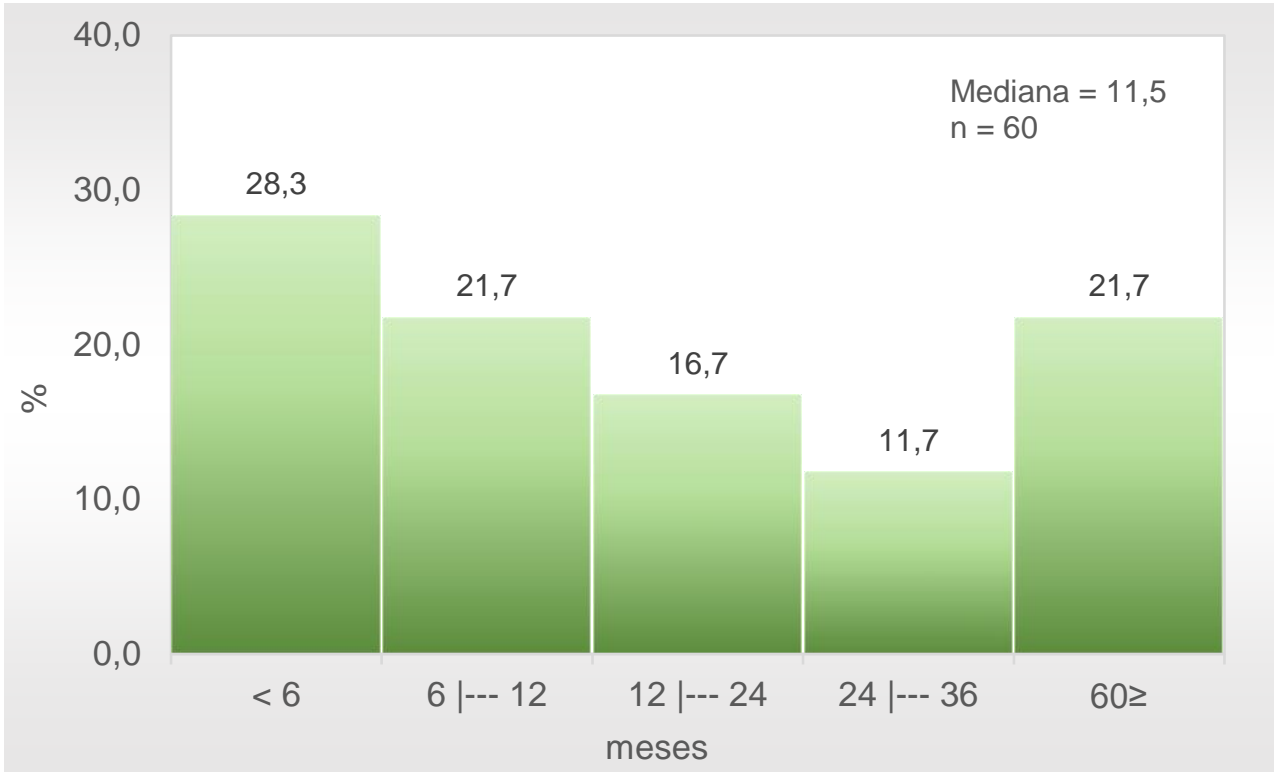

Fonte: Maia CP, et al., 2021.

Quando as participantes da pesquisa foram questionadas sobre a alteração do padrão de sangramento, a queixa mais prevalente foi a de aumento significativo do fluxo da menstruação (50\%), sangramento forte (35\%) com necessidade do uso de absorvente noturno (41,7\%) e eliminação de grandes coágulos (66,7\%). Das pacientes estudadas, $61,7 \%$ relataram já terem apresentado sintomas como fraqueza, perca do fôlego ou sensação de palpitações durantes os episódios de sangramento, tendo necessitado de atendimento emergencial para controle da hemorragia (Tabela 2).

Tabela 2 - Distribuição segundo a caracterização do sangramento das mulheres com SUA atendidas em ambulatório de ginecologia da cidade de Manaus.

\begin{tabular}{lcc}
\hline Variáveis $(\mathbf{n}=\mathbf{6 0})$ & Frequência absoluta simples & $\%$ \\
\hline Tipo de sangramento & & \\
\hline Aumento no no de dias da menstruação & 23 & 38,3 \\
Aumento no fluxo da menstruação & 30 & 50,0 \\
Entre as menstruações & 17 & 28,3 \\
Sem parar & 18 & 30,0 \\
\hline Fluxo do sangramento & 9 & 15,0 \\
\hline Leve & 11 & 18,3 \\
Moderado & 21 & 35,0 \\
Forte & 19 & 31,7 \\
$\quad$ Muito forte & 22 & 36,7 \\
\hline Tipo de absorvente & 12 & 20,0 \\
\hline Comum & 25 & 41,7 \\
Fralda & 1 & 1,7 \\
$\quad$ Noturno & 40 & 66,7 \\
$\quad$ Interno & 37 & \multirow{2}{*}{61,7} \\
\hline Elimina grandes coágulos & 37 & 61,7 \\
\hline Já se sentiu fraca, sem fôlego ou com & 37 \\
palpitações durante o período de sangramento & & \\
\hline Já procurou o serviço de emergência durante o & & \\
sangramento & & \\
\hline
\end{tabular}

Fonte: Maia CP, et al., 2021. 
Quanto às questões referentes à relação entre os episódios de SUA e a qualidade de vida, mais da metade das mulheres classificaram-na entre regular e muito ruim (56,7\%). Foi referido por $46,7 \%$ delas que o quadro interfere continuamente no dia a dia e na vida social, por causa das limitações diversas decorrentes do sangramento e do mal-estar que o acompanha. Mais especificamente o SUA foi apontado com causador de interferências constantes na qualidade do sono (31,7\%), na prática de exercícios físicos $(38,3 \%)$, na vida sexual $(58,3 \%)$ e no desempenho laboral (35\%) (Tabela 3).

Tabela 3 - Distribuição segundo os os indicadores de qualidade de vida das mulheres com SUA atendidas em ambulatório de ginecologia da cidade de Manaus.

\begin{tabular}{|c|c|c|}
\hline Variáveis $(n=60)$ & Frequência absoluta simples & $\%$ \\
\hline \multicolumn{3}{|c|}{ Como avalia a qualidade de vida } \\
\hline Muito ruim & 3 & 5,0 \\
\hline Ruim & 9 & 15,0 \\
\hline Regular & 22 & 36,7 \\
\hline Boa & 21 & 35,0 \\
\hline Muito boa & 5 & 8,3 \\
\hline \multicolumn{3}{|c|}{ O sangramento interfere no dia a dia, na vida social } \\
\hline Quase nunca & 2 & 3,3 \\
\hline Nunca & 9 & 15,0 \\
\hline Às vezes & 21 & 35,0 \\
\hline Sempre & 28 & 46,7 \\
\hline \multicolumn{3}{|c|}{ O sangramento interfere na qualidade do sono } \\
\hline Quase nunca & 2 & 3,3 \\
\hline Nunca & 9 & 15,0 \\
\hline Às vezes & 30 & 50,0 \\
\hline Sempre & 19 & 31,7 \\
\hline \multicolumn{3}{|c|}{ O sangramento interfere na prática de atividade física } \\
\hline Quase nunca & 5 & 8,3 \\
\hline Nunca & 15 & 25,0 \\
\hline Às vezes & 17 & 28,3 \\
\hline Sempre & 23 & 38,3 \\
\hline \multicolumn{3}{|c|}{ O sangramento interfere na vida sexual } \\
\hline Quase nunca & 2 & 3,3 \\
\hline Nunca & 11 & 18,3 \\
\hline Às vezes & 12 & 20,0 \\
\hline Sempre & 35 & 58,3 \\
\hline \multicolumn{3}{|c|}{ O sangramento interfere no emprego } \\
\hline Quase nunca & 2 & 3,3 \\
\hline Nunca & 19 & 31,7 \\
\hline Às vezes & 18 & 30,0 \\
\hline Sempre & 21 & 35,0 \\
\hline Quase nunca & 2 & 3,3 \\
\hline
\end{tabular}

Fonte: Maia CP, et al., 2021.

\section{DISCUSSÃO}

Das múltiplas intercorrências na saúde da mulher, o sangramento uterino anormal (SUA) é condição frequente no atendimento ginecológico acometendo grande parte da população feminina, em diferentes momentos da vida. A literatura sugere que aproximadamente $30 \%$ das pacientes na menacme procuram 0 ginecologista para tratamento desta afecção, porém segundo Sousa GB, et al. (2019), a prevalência de SUA no Amazonas é de $22 \%$, estando acima da encontrada em países subdesenvolvidos (5 a 15\%). Em nosso 
estudo a faixa etária mais prevalente foi entre 40 e 50 anos de idade. Este dado também é confirmado por Sousa GB, et al. (2019), que mostrou que o maior percentual de mulheres amazonenses atendidas ambulatorialmente com quadro de SUA apresentavam idade acima de 35 anos.

Nesse contexto, é possível observar a influência da escolarização e demais elementos de formação pessoal além de fatores culturais no entendimento do diagnóstico e no comprometimento com o tratamento proposto, a fim de garantir resultados favoráveis e atingir às expectativas dessas mulheres. Isto foi corroborado pelo fato da maioria das pacientes atendidas possuírem nível médio de escolaridade, o que é vantajoso ao considerarmos que quanto maior o entendimento dos fatores concorrentes para o binômio doença-saúde, melhor tende a ser a compreensão da enfermidade e o engajamento no tratamento para superação desta condição (TAVARES NUL, et al., 2016).

Nahidi FA, et al. (2011) em estudo de caso controle, correlacionou a via de parto com o SUA e identificou que os distúrbios menstruais eram mais comuns nas mulheres com histórico de cesariana. Porém, esse dado é divergente dos nossos achados que mostram uma maior prevalência de histórico de parto por via vaginal nas pacientes com sangramento anormal $(58,3 \%)$ quando comparado com partos por cesariana $(31,7 \%)$. Essa discordância pode se dar devido a questões culturais do Amazonas que tendem a favorecer o parto normal frente ao parto por via alta.

A quantidade de parceiros sexuais e o início precoce da atividade sexual se apresentam como importantes fatores de risco para infecções sexualmente transmissíveis (IST) as quais apresentam quadro clínico variável podendo se manifestar inclusive através de sangramento vaginal. Em nosso estudo $23,3 \%$ das pacientes relataram primeira relação sexual antes dos 15 anos de idade e $40 \%$ referiram terem tido entre 2 e 5 parceiros sexuais. Logo, é importante que as ISTs sejam consideradas como diagnóstico diferencial na investigação do SUA (KAUNITZ AM, 2021).

No Amazonas o acesso aos serviços de saúde apresenta grande desigualdade entre as cidades do interior e Manaus. A extensão territorial e a insuficiente disponibilidade de recursos humanos e materiais determinam a clara centralização dos serviços de maior complexidade na capital do Estado, o que muitas vezes dificulta e atrasa a busca por assistência especializada. Esse argumento explica a pequena taxa de mulheres atendidas advindas do interior do Amazonas (13,3\%) em comparação com as procedentes da capital (86,7\%). Entretanto, o pequeno percentual de pacientes interioranas não permite inferirmos que o SUA seja condição pouco recorrente nesta população, apenas que a disponibilidade de atendimento ginecológico in loco é mais escassa (GARNELO L, et al., 2017).

Nesta análise a maior parte da amostra de pacientes se autodeclarou como parda, o que pode estar relacionado com uma maior prevalência desta raça no Amazonas, considerando a grande miscigenação que caracteriza a população amazônica (INSTITUTO BRASILEIRO DE GEOGRAFIA E ESTATÍSTICA, 2011). Entretanto, o SUA é condição fisiopatológica que atinge mulheres de forma geral, independente de raça ou outras especificações que as diferenciem, uma vez que as características do ciclo menstrual são genéricas.

O ciclo menstrual, segundo a literatura, apresenta duração média de vinte e oito dias. O tempo de sangramento varia de três a oito dias e o volume médio desprendido entre cinco e oitenta ml (KAUNITZ AM, 2021). Entretanto, é sabido que o padrão de sangramento normal é individualizado, enquanto o SUA pode ser diagnosticado por meio de mudanças na intensidade, duração e frequência do sangramento (ARLINDO EM et al., 2018).

Não há método específico e preciso para quantificação do volume de sangue eliminado, por isso na pesquisa usou-se como referência a autopercepção, que apresenta sensibilidade de $74 \%$ no diagnóstico de sangramento aumentado. Para melhor clareza dos eventos de sangramento das mulheres estudadas, também foi indagado o tipo de absorvente usado nessas ocorrências, se há eliminação de grandes coágulos e se já houve eventos que podem ser indicativos clínicos de anemia associados ao sangramento como como astenia ou taquicardia. Correlacionando as variáveis referentes a intensidade do sangramento, verificou-se que $66 \%$ da população estudada apresentou fluxo menstrual aumentado. Essa caracterização permite uma interpretação objetiva quanto aos resultados clínicos de intervenções terapêuticas (MAGNAY JL, et al., 2018). 
A qualidade de vida é um dos principais objetivos pretendidos pela medicina na atualidade. A satisfação pessoal é cada vez mais valorizada em detrimento do prolongamento da vida em condições incapacitantes. Nobre RCN (1995) aborda qualidade de vida como sensação íntima de conforto, bem-estar ou felicidade no desempenho de funções físicas, intelectuais e psíquicas dentro da realidade da sua família, do seu trabalho e dos valores da comunidade à qual pertence. Assim, devido a subjetividade e multidimensionalidade do tema, a avaliação da qualidade de vida é inerente a cada indivíduo, não podendo ser estabelecida por terceiros. Por meio deste conceito podemos ressaltar o impacto em mais da metade da população estudada, quando se avalia o sangramento. Além disso, Aguas F, et al. (2019) apontam que as queixas relacionadas à qualidade de vida apresentadas pelas mulheres com SUA podem estar relacionadas tanto ao sangramento em si quanto aos níveis de hemoglobina e hematócrito da paciente.

A pesquisa destacou a significativa repercussão na qualidade de vida das pacientes, pelo comprometimento da sensação de bem-estar devido às implicações diretas e indiretas do SUA. A necessidade de uso e trocas constantes de absorventes, as interferências na atividade sexual, na prática de exercícios e a ansiedade por possíveis constrangimentos em ocasiões públicas são apenas alguns exemplos de prejuízos ao bem-estar das mulheres (BUSHNELL DM, et al., 2010). Neste estudo 56,7\% das pacientes classificaram sua qualidade de vida entre regular e muito ruim e $46,7 \%$ referiram que o sangramento sempre interfere diretamente no dia a dia e na vida social de alguma forma, comprovando o impacto do SUA na percepção geral de bem-estar relatado na literatura (CLARK TJ, et al., 2012; SEIDL EMF e ZANNON CMLC, 2004).

Estudos sugerem a importância da percepção positiva de qualidade de vida em associação a diversos processos relacionados à saúde, não apenas no âmbito fisiológico, mas também no comportamental e cognitivo. Isto se justifica pela grande relevância que a dimensão emocional tem sobre os aspectos patológicos, notadamente nos casos crônicos ou de maior duração. Inferimos então, que a avaliação de indicadores de bem-estar, qualidade de vida e saúde são elementos bastante importantes a serem considerados quando da tomada de decisões referentes às possíveis intervenções medicamentosas ou hospitalares (MACHADO WL e BANDEIRA DR, 2012).

Bruinvels G, et al. (2016) analisou a prevalência e as consequências do SUA em mulheres que praticam atividade física regularmente e demostrou que esta disfunção acomete mais de um terço dessa população e com frequência está associado a deficiência de ferro, podendo resultar em fadiga, fraqueza, cognição prejudicada e morbidade psicológica. Nosso estudo corrobora estes dados, pois $38,3 \%$ das pacientes referiram sangramento que sempre interfere na prática de atividade física.

É sabido que a sexualidade feminina sofre mais influência de fatores psicológicos e sociais que a masculina, e que há escassez de estudos correlacionando os aspectos diversos da sexualidade com o SUA. Uma parcela muito significativa das mulheres $(78 \%)$ referiu que o sangramento impacta negativamente sua vida sexual com alguma frequência. Um estudo realizado em São Paulo demostrou que as pacientes com SUA apresentam um risco aumentado de desenvolver disfunção sexual, uma vez que suas capacidades funcional, emocional e social se encontram amplamente comprometidas levando a interferências e restrições da atividade sexual (FRANCESCHINA BEB, et al., 2019).

A diminuição da frequência e do interesse sexual decorrentes do SUA podem levar ao desenvolvimento de quadros de depressão e ansiedade, por ser potencial agressor da feminilidade, devido à redução da libido e da autoestima feminina. O SUA pode também potencializar a Síndrome da Tensão Pré-Menstrual, ampliando ainda mais as desordens emocionais relacionadas às modificações físicas e subjetivas, com possíveis consequências nos relacionamentos interpessoais e envolvendo o desempenho laboral (ARLINDO EM, et al., 2018).

Além do impacto direto no bem-estar feminino, o SUA também está associado a elevados custos econômicos direta ou indiretamente. Seja nos recursos investidos pela paciente e pelo sistema de saúde no tratamento, seja interferindo na produtividade diária e no ambiente de trabalho. É sabido que as mulheres que apresentam sangramento irregular têm maior número de ausências ao trabalho, além disso, são vistas em apenas $72 \%$ dos cenários profissionais quando comparadas às mulheres com sangramento normal (CÔTÉ I, 
et al., 2012). Em nossa pesquisa $65 \%$ das pacientes referiram interferência direta do sangramento anormal nas suas atividades laborais.

\section{CONCLUSÃO}

Considerando os dados obtidos, foi possível observar que o impacto do sangramento na qualidade de vida das mulheres estudadas - notadamente o aumento de volume do fluxo menstrual, foi característica marcante, especialmente no âmbito da vida sexual e social. Os achados possibilitaram o conhecimento do perfil clínico e epidemiológico, bem como a percepção de que os constantes incômodos associados a desordens emocionais, físicas, sexuais e laborais têm impedido as pacientes com SUA de se sentirem plenamente atendidas em relação às suas expectativas de qualidade de vida. Tais aspectos devem ser levados em consideração a fim de proporcionar uma abordagem integral, resolutiva e individualizada que ofereça condições mais viáveis ao bem-estar. Estudos futuros com populações com características sociodemográficas e ambientais semelhantes poderão fortalecer esta casuística.

\section{REFERÊNCIAS}

1. AGUAS F, et al. Tratamento da Anemia ferropenica: Recomendações da Sociedade Portuguesa de Ginecologia. Acta Obstet Ginecol Port, 2019; 13: 56-61.

2. ARLINDO EM, et al. Telecondutas: Sangramento uterino anormal. Porto Alegre: Telessaúde RS-UFRGS, 2018.

3. BENETTI-PINTO CL, et al. Abnormal uterine bleeding. Rev. Bras. Ginecol. Obstet., 2017; 39: 358-368.

4. BRUINVELS G, et al. Heavy menstrual bleeding in exercising females. Plos One, 2016; 11(2): e0149881.

5. BUSHNELL DM, et al. Menorrhagia impact questionnaire: assessing the influence of heavy menstrual bleeding on quality of life. Current Medical Research \& Opinion, 2010; 26(12): 2745-2755.

6. CLARK TJ, et al. Quality of life instruments in studies of menorrhagia: a systematic review. European Journal of Obstetrics \& Gynecology and Reproductive Biology, 2012; 104: 96-104.

7. CÔTÉ I, et al. Work loss associated with increased menstrual loss in the United States. The American College of Obstetricians and Gynecologists, 2012; 100(4): 683-687.

8. FILHO ALS. Sangramento uterino anormal: proposta de abordagem do GrupoHeavy Menstrual Bleeding. Femina, 2015; 43(4): 161-166.

9. FRANCESCHINA BEB, et al. Avaliação da sexualidade em mulheres com sangramento uterino anormal. Revista dos Trabalhos de Iniciação Científica da UNICAMP, 2019; 27: 32-33.

10. FRASER IS, et al. Abnormal uterine bleeding reproductive-age women: Terminology and PALM-COEIN etiology classification. UpToDate, 2018.

11. GARNELO L, et al. Regionalização em Saúde no Amazonas: avanços e desafios. Ciência \& Saúde Coletiva, 2017; 22(4):1225-1234

12. INSTITUTO BRASILEIRO DE GEOGRAFIA E ESTATÍSTICA. Indicadores Sociais Municipais: uma análise dos resultados do universo do censo demográfico 2010. Rio de Janeiro: IBGE, 2011; 149p.

13. KAUNITZ AM. Approach to abnormal uterine bleeding in nonpregnant reproductive-age women. UpToDate, 2021.

14. MACHADO WL, BANDEIRA DR. Bem-estar psicológico: definição, avaliação e principais correlatos. Estudos de psicologia, 2012; 29(4): 587-595.

15. MAGNAY JL, et al. A systematic review of methods to measure menstrual blood loss. BMC Women's Health, 2018; 18(1): 142-155.

16. MUNRO M, et al. The FIGO classification of causes of abnormal uterine bleeding in the reproductive years. Fertility and Sterility, 2011; 95(7): 2204-2208.

17. NAHIDI FA, et al. Relationship between delivery type and menstrual disorders: A case-control study. JRHS 2011; 11(2): 83-90.

18. NOBRE RCN. Qualidade de vida. Arq Bras Cardiol, 1995: 64(4): 299-300.

19. SEIDL EMF, ZANNON CMLC. Qualidade de vida e saúde: aspectos conceituais e metodológicos. Cad. Saúde Pública, 2004; 20(2): 580-588.

20. SILVA NK. Abnormal uterine bleeding in adolescents: Evaluation and approach to diagnosis. UpToDate, 2021.

21. SINGH S, et al. Abnormal Uterine Bleeding in Pre-Menopausal Women. Journal of Obstetrics and Gynaecology Canada, 2018; 40(5): 391-415.

22. SOUSA GB, et al. Estudo da prevalência de sangramento uterino anormal na Amazônia Ocidental: aspectos clínicos e epidemiológicos. Revista Eletrônica Acervo Saúde, 2019; 11(15): e1287.

23. TAVARES NUL, et al. Fatores associados à baixa adesão ao tratamento farmacológico de doenças crônicas no Brasil. Revista de Saúde Pública, 2016; 50(2): 1-11.

24. VIEIRA S. Bioestatística, Tópicos Avançados. 2a ed. Rio de Janeiro: Elsevier, 2004; 216p.

25. YELA DA, BENETTI-PINTO CL. Sangramento uterino anormal - Protocolos Febrasco №42. São Paulo: Federação Brasileira das Associações de Ginecologia e Obstetrícia, 2018; 20p. 